POS PROCEEDINGS

\title{
DBBC3: VLBI at 32 Gbits per second
}

\section{Gino Tuccari, Salvo Buttaccio}

INAF - Istituto di Radioastronomia, Contrada Renna Bassa, I-96017 Noto, Italy

E-mail: g.tuccarieira.inaf.it

Walter Alef*, Michael Wunderlich, David A. Graham, Alessandra Bertarini, Alan Roy, Jan Wagner

Max-Planck-Institut für Radioastronomie, Auf dem Hügel 69, D-53121 Bonn, Germany

E-mail: walef@mpifr-bonn.mpg.de

\section{Gianni Comoretto}

INAF - Osservatorio Astrofisico di Arcetri, Largo E. Fermi 5, I-50125 Firenze, Italy

\section{Michael Lindqvist, Simon Casey}

Onsala Space Observatory, SE-439 92 Onsala, Sweden

\begin{abstract}
The project to develop the third generation of a digital backend system (DBBC3) for VLBI and other applications is presented, including an overview of the evolution of this system. The initial development started about ten years ago and evolved in the course of time by improving all its components: hardware, firmware and software going from DBBC1 to DBBC2. The latest, now third generation will be able to fully implement digitally all the functionality required of a complete $32 \mathrm{Gbps}$ VLBI backend for the EVN and VLBI2010. The architecture and adopted methods are described. The implementation of the astronomical version is financially supported by RadioNet3.
\end{abstract}

11th European VLBI Network Symposium \& Users Meeting,

October 9-12, 2012

Bordeaux, France

\footnotetext{
* Speaker.
} 


\section{Introduction}

The development of the DBBC started in 2004 [1, 2]. During the previous few years ad-hoc laboratory experiments and experiments with real sky signals had indeed demonstrated the possibility of emulating with fully digital equipment the entire functionality of the MK4 VLBI analogue terminal. In the digital process the intermediate-frequency signal available from the receiver is immediately converted to a digital representation. Before about 2004 this complete digital process could not be fully implemented at a reasonable cost, and moreover it was a technical challenge due to the the wide band and the high frequencies involved. During the first decade of 2000 with progressive improvements the DBBC project evolved to cope with an input bandwidth of up to $4 \times 1 \mathrm{GHz}$.

The first DBBC version (DBBC1; 2004 - 2008) [3] was a backwards-compatible replacement of the existing VLBI terminal, while with the DBBC2 (2007 to date), additional observing modes became available which did not exist in the analogue backend. The further enhanced version of the DBBC2 for VLBI2010 [4], the DBBC2010 (2009 to date) is compatible with the proposed VLBI2010 observing mode.

One way to increase the sensitivity of a VLBI network is to increase the observing bandwidth. With new wide-band receivers the demand for backends which can handle bandwidths of several $\mathrm{GHz}$ has arisen. Also the EVN has been increasing its maximum data rate from a maximum of 1 Gbps with the MK4 analogue backend to a maximum of 4 Gbps with the present DBBC2 - a data rate which is under test in the EVN now.

In preparation for receivers and IF systems which will deliver $4 \mathrm{GHz}$ or more bandwidth to the backends it was felt necessary to develop a system which can process an instantaneous bandwidth of $4 \mathrm{GHz}$ per polarization as a minimum. The resulting output data rate for a dual polarisation receiver is then at least $32 \mathrm{Gbps}$, with the option of 64 Gbps for a 4 IF system. Such a backend is the intermediate goal of the DBBC3 project.

The specifications of VLBI2010 define a set of features of the receiving/backend system to achieve the goal of greatly improved geodetic measurement precision. The telescopes will operate in a single broad band ranging from 2 to $14 \mathrm{GHz}$ observing in dual linear polarization. Inside this frequency range a subset of four $1024 \mathrm{MHz}$ wide pieces will be selected, in both polarizations. This will realise bandwidth synthesis, the fitting of phase slopes over a wide frequency range for a much wider portion of the spectrum than is possible with the present system. Such a wide input band could also be of great interest for astronomy because of the significant increase in sensitivity. Being able to process an entire $14 \mathrm{GHz}$ wide piece of band could be a quantum leap in digital radio astronomy data acquisition. This goal is very ambitious and its implementation in a radio astronomy backend would be a novelty. To digitally sample and process the whole $14 \mathrm{GHz}$ wide band or a number of sub-bands thereof is the final goal for the DBBC3 project.

\section{DBBC3 Structure}

The DBBC3 system needs to meet stringent requirements: it has to be backwards compatible with the existing legacy backends and has to be able to realise the new functionality in a wideband environment. Moreover it should be able to accomplish all the required functionalities for the 
planned EVN goals (min $2 \times 4 \mathrm{GHz}$ bandwidth) and VLBI2010 $(2 \times 14 \mathrm{GHz}$ bandwidth). As many stations are active in both networks a single system is mandatory. Flexibility is a requirement due to the different radiotelescopes and their dissimilar receivers and IF systems in terms of number and type of IFs.

To be compatible with the existing systems, the new hardware needs to be mechanically and electrically level-compatible. This aspect is useful because existing DBBC terminals in the field could be upgraded to meet the new performance requirement by replacing the necessary parts. Components of the DBBC3 can be inserted in existing DBBC2 and DBBC2010 backends to augment their performance with additional features.

The much higher performance of the new backends requires new hardware parts, to be accompanied by new firmware. A clear development path has been laid out to minimise risks in the project. In a first step a DBBC3-L will be developed which can be seen as a fully qualified $4 \mathrm{GHz}$ $\mathrm{DBBC}$ and at the same time will allow us to study how best to achieve the final goal of a $14 \mathrm{GHz}$ DDBC3-H.

The main features of the DBBC3-L system are:

- Maximum number of wide input IFs: 4

- Instantaneous bandwidth in each IF : $4 \mathrm{GHz}$

- Sampling representation: 10 bit

- Processing capability N x 5 TMACS (multiplication-accumulation per second), with $\mathrm{N}$ the number of processing nodes

- Output data rate: max $64 \mathrm{Gbps}$

- Compatibility with the existing DBBC environment.

The main features of the DBBC3-H system are:

- Max number of wide input IFs: 4

- Instantaneous bandwidth in each IF : $14 \mathrm{GHz}$

- Sampling representation: 8 bit

- Processing capability N x 5 TMACS (multiplication-accumulation per second), with $\mathrm{N}$ the number of processing nodes

- Output data rate: max 896 Gbps

- Compatibility with the existing DBBC environment.

In this paper we will describe mainly the DBBC3-L version which is being specifically developed for the EVN network and is supported by RadioNet3. In Figures 1,2 and 3 are shown the main schematic components of the DBBC3-L: the overall architecture, the ADB3-L structure, the CORE3-L structure, and the FILA40G concept.

The structure of the system is straightforward. Four IFs $4 \mathrm{GHz}$ wide are sampled with 10-bit representation. This data is then transferred to one or more dedicated processing nodes, with their own single element identity and functionality. The processors then extract from the digital data 


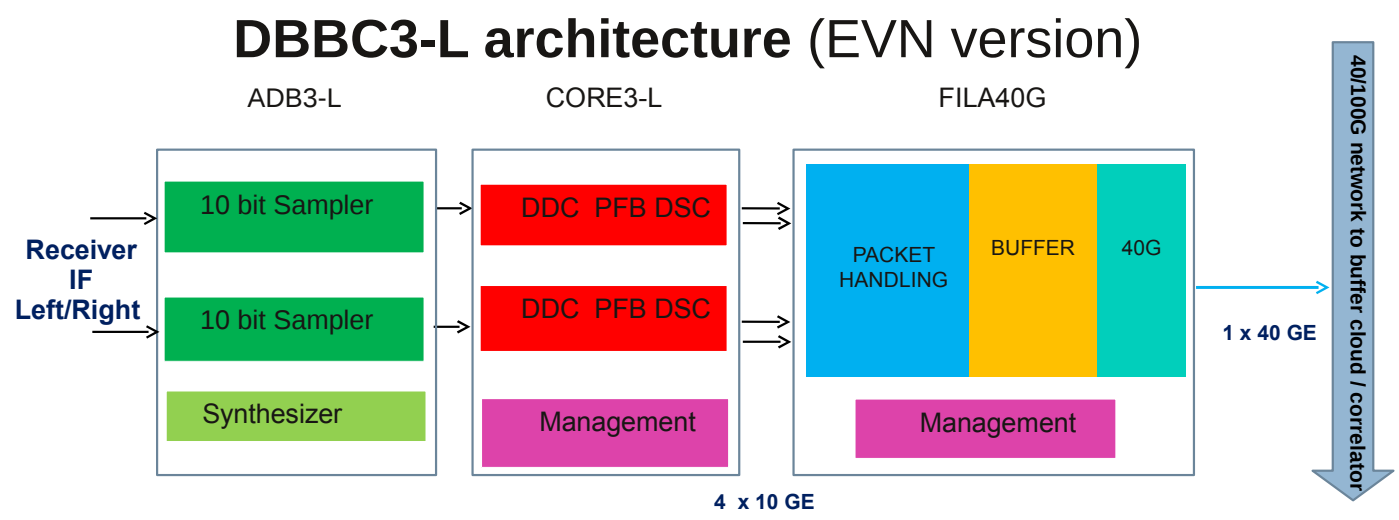

Figure 1: DBBC3-L block diagram. The system consists of an analogue to digital converter "ADB3-L", the processing unit with a powerful FPGA "CORE3-L", and the unit responsible for transporting the processed and formatted data onto 40/100 Gb Ethernet "FILA40G".
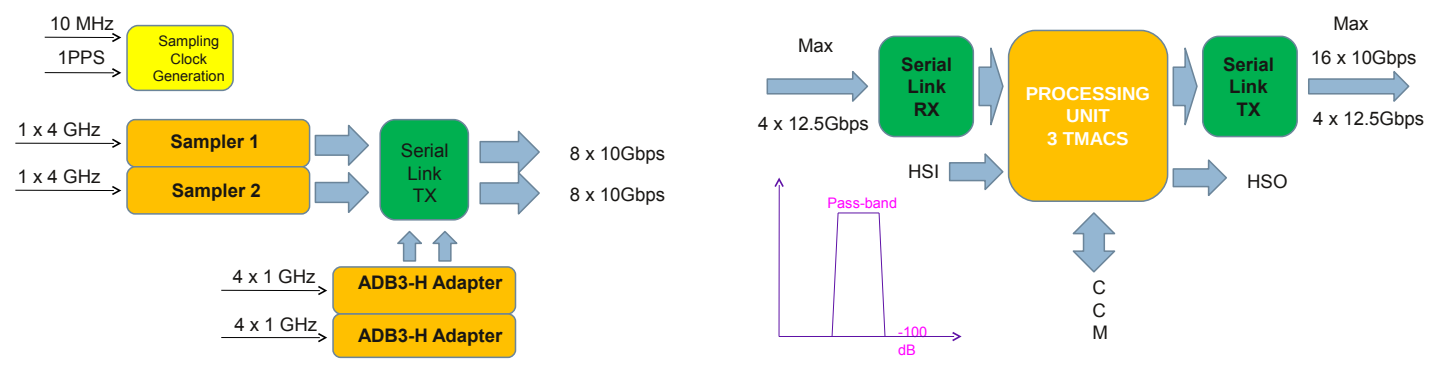

Figure 2: Block diagrams of the analogue-digital converter ADB3-L (left) and the processing unit CORE3L (right). "HSI" is the highspeed input bus, "HSO" the high speed output bus, and "CCM" the command, control and monitoring bus.

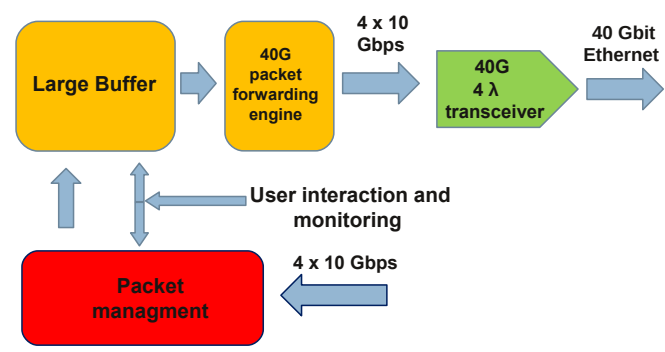

Figure 3: Block diagrams of the FILA40G unit. The data comes into the "packet management" subunit as $4 \times 10 \mathrm{Gbps}$ data streams. This subunit will handle additional data processing. From there via the "large buffer" the data flows through the "40G packet forwarding engine" to a 40GE transceiver and on to Ethernet. 
streams portions of the band (with digitally tunable mixers or fixed filters) and produce VLBIcompatible output VDIF (VLBI Data Interchange Format) packets .

The last element of the chain is the FILA40G subunit whose function is to condense the data onto single optical fibres at $40 \mathrm{Gbps}$ data rate and to handle the data at network packet level. A dedicated version, the FILA40G-ST, will in addition have storage capabilities.

\subsection{ADB3-L Sampler}

The massive sampling is performed by state of the art sampler chips. An extensive analysis is under way to determine the phase performance of these devices, as the interferometric application calls for high absolute phase stability and low temporal jitter. An alternative general method to increase the bandwidth is to make use of complex samples: two channels in quadrature are sampled at a clock frequency equal to the full instantaneous bandwidth. A single ADB3-L has on-board four complete samplers, with the possibility to arrange them for a variety of features, single and multiple, real or complex. Indeed for example in real mode the four samplers can be fed with a single input signal to operate on the full $4 \mathrm{GHz}$ or with two signals each of $2 \mathrm{GHz}$ instantaneous bandwidth, or finally with four signals of $1 \mathrm{GHz}$ bandwidth.

Sampled data have to be transferred to the processing stage, and due to the high data rate of $64 \mathrm{Gbps}$ a parallel bus cannot be implemented owing to the very large number of differential lines required and the high operation frequency. Pre-processing is used to transfer this large data rate to a manageable number of serial connections to link the Sampler with the Processing unit.

\subsection{CORE3-L Processing Node}

Data coming from the sampler board ADB3-L are routed to the processing node CORE3 using the lanes of the high speed input bus. This board is able to process data in different ways: DSC (Direct Sampling Conversion), DDC (Digital Down Converter) and PFB (Polyphase Filter Bank) functionalities. Additional capabilities will support spectroscopic and polarimetric observations.

From the pool of channels a subset is selected according to the desired output data rate defined by the observer or allowed by the recording or network media. The data is output through the high speed output bus. Additional input and output connections are available to maintain compatibility with the DBBC2 stack.

The large processing resources available in the FPGA chosen for the CORE3-L allows implementation of digital filters in the class of $100 \mathrm{~dB}$ for in/out band rejection. This feature is required for the expected presence of large RFI signals in the very wide input band. This excellent discrimination together with the tuning ability should be appropriate to obtain useful down-converted and clean slices of the observed band.

As an alternative input the board will be able to receive data packets from a block of ADB3$\mathrm{H} / \mathrm{CORE} 3-\mathrm{H}$ units to be routed to the rest of the system for additional data processing.

\subsection{FILA40G Network Node}

Data from the converted bands are finally transferred to the network controller FILA40G as multiple 10GE connections. The number of connections is then accumulated into a 40GE data stream to be transferred to the final destination points. Such final points could be recorders, nodes 
of VLBI correlators or a buffer cloud. In addition to the 40G network capability the FILA40G unit will be able to manipulate the data packets to perform functions like corner-turning, pulsar-gating, packet filtering and routing, burst mode accumulation, and others that could be required at the packet level as VLBI methods evolve. In addition a dedicated version will be provided which can include storage elements for data buffering and recording.

\section{Preliminary Results}

The DBBC3-L project is progressing as planned and the first prototypes are under development and construction. These will produce the proper information and know-how to proceed to the final version. The evaluation performed in the laboratory until now shows that the project will reach the planned goals in the scheduled time without major risks or problems, despite the very challenging performance to be obtained.

Tests and experiments performed with the ADB3-H prototypes are also already available and show that direct data conversion to the digital domain for the full $14 \mathrm{GHz}$ band is possible without the need for an initial analogue down-conversion. This represents a huge, challenging and intriguing step ahead in the simplification and improvement of the VLBI2010 electronics which should significantly reduce the system cost. The term "backend" will no longer be appropriate due to the typical frontend functionality this system incorporates. The EVN network will also greatly benefit in the near future by this quantum leap in sensitivity.

\section{Acknowledgments}

The research leading to these results has received funding from the European Commission Seventh Framework Programme (FP/2007-2013) under grant agreement No 283393 (RadioNet3).

\section{References}

[1] G. Tuccari, Development of a Digital Base Band Converter (DBBC): Basic Elements and Preliminary Results, in: New Technologies in VLBI, Astronomical Society of the Pacific Conference Series, ISSN 1050-3390, Vol. 306, 177-252, 2004.

[2] G. Tuccari, A.R. Whitney, H. Hinteregger, Y. Koyama, T. Kondo, Backend Systems-Perspectives in 2010, in: International VLBI Service for Geodesy and Astrometry 2004 General Meeting Proceedings, N.R. Vandenberg and K.D. Baver (eds), NASA/CP-2004-212255, pp. 80-84, 2004.

[3] G. Tuccari, DBBC - a Wide Band Digital Base Band Converter in: International VLBI Service for Geodesy and Astrometry 2004 General Meeting Proceedings, N.R. Vandenberg and K. D. Baver (eds), NASA/CP-2004-212255, pp. 234-237, 2004.

[4] A.E. Niell, A.R. Whitney, B. Petrachenko, W. Schlüter, N.R. Vandenberg, H. Hase, Y. Koyama, C. Ma, H. Schuh, and G. Tuccari, VLBI2010: Current and Future Requirements for Geodetic VLBI Systems, in: International VLBI Service for Geodesy and Astrometry 2005 Annual Report, D. Behrend and K. Baver (eds.), NASA/TP-2006-214136, pp. 13-40, 2006. 